were able to cross the midline, whereas in rescued robo mutants, Robo was localized to dendrites that were repelled by the midline.

These findings raise an intriguing question about the regulation of Robo and Frazzled expression in developing neurons. Many neurons extend several axonal and dendritic processes, of which some project contralaterally and some ipsilaterally. Together with the observations on the subcellular localization of Robo and Frazzled in rescued mutant neurons, this indicates that Robo and Frazzled are probably targeted differentially to processes that emanate from the same cell. The question of how this is achieved will undoubtedly provide a focus for future investigations.

Heather Wood

(4) References and links ORIGINAL RESEARCH PAPER Furrer, M.-P. et al. Robo and Frazzled/DCC mediate dendritic guidance at the CNS midline. Nature Neurosci. 6 , 223-230 (2003)

FURTHER READING Yu, T. W. \& Bargmann, C. I. Dynamic regulation of axon guidance. Nature Neurosci. 4, 1169-1176 (2001)

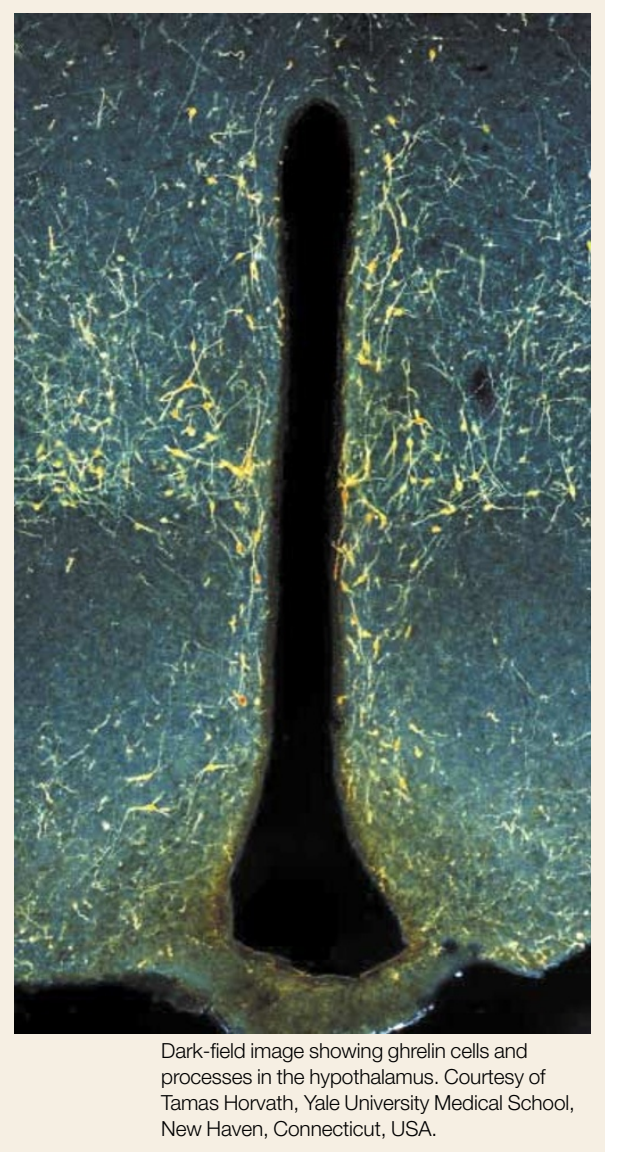

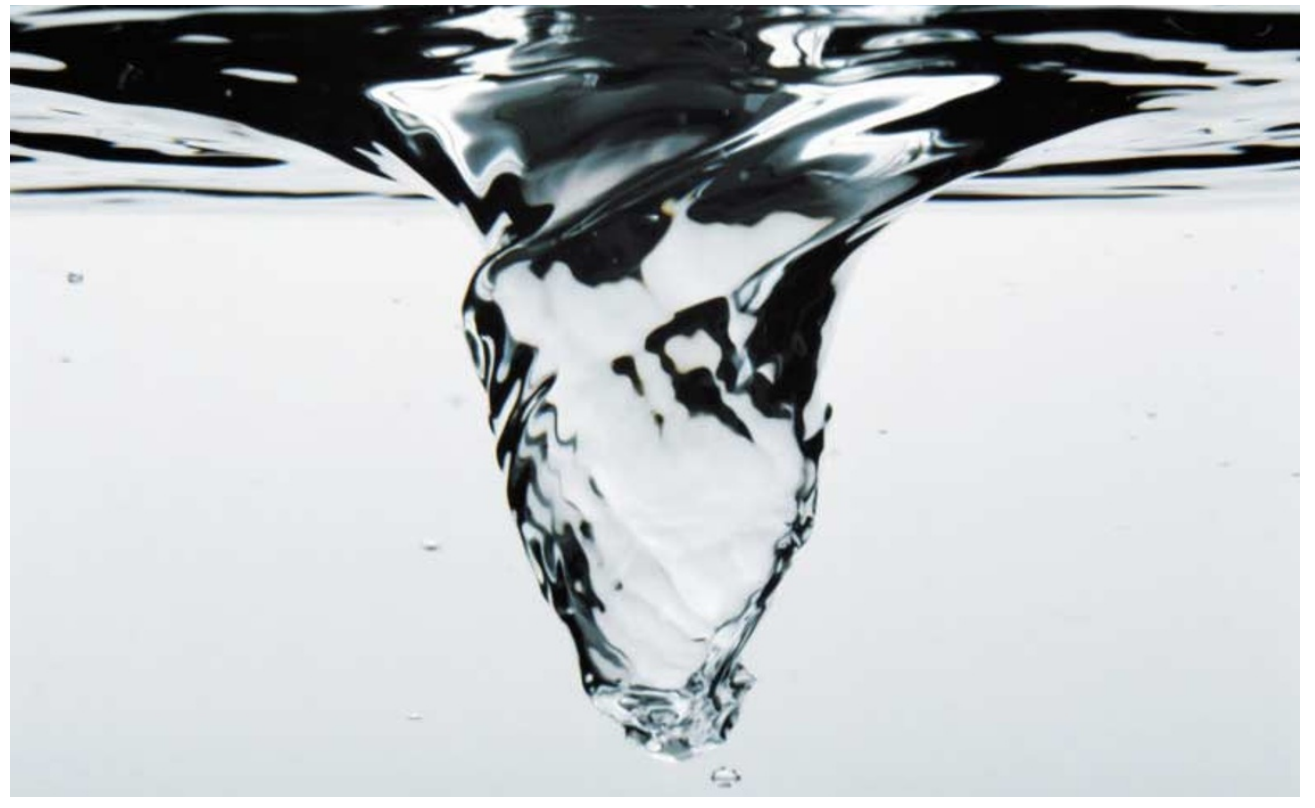

BLOOD-BRAIN BARRIER

\title{
Water in the brain
}

In the one-week period after a stroke, cerebral oedema - swelling of brain tissue due to the rapid influx of water - is one of the factors most likely to cause death. The development of efficient therapies for this condition has been hampered by a lack of information regarding the route of water flow into the brain. But new data from AmiryMoghaddam and colleagues implicates the water channel AQP4 in water flow across the interface between blood and brain.

AQP4 is preferentially localized to the astrocyte membrane domains that form the interface between the brain and the extracerebral liquid spaces (which comprise the cerebral microvessels and subarachnoidal space). AQP4 is anchored in these domains by an adaptor protein, $\alpha$-syntrophin - knocking out the expression of $\alpha$-syntrophin in mice decreases the density of AQP4 in these brain regions at least eight-fold. Using $\alpha$-syntrophin knockout mice, AmiryMoghaddam et al. temporarily occluded the middle cerebral artery to induce ischaemia. After reperfusion of the occluded artery, the volume of cerebral oedema was significantly reduced relative to wild type, indicating that water influx through AQP4 is a key regulator of ischaemiainduced oedema.

As AQP4 is thought to facilitate the bidirectional movement of water, the authors wondered if its capacity to facilitate water efflux from the brain influenced the rate at which swelling dissipates following ischaemic insult. This point is of particular therapeutic interest as swelling is slow to subside after stroke, significantly prolonging the risk of long-term damage. Immunolabelling of wild-type brains using anti- $\mathrm{AQP} 4$ antibodies revealed that $\mathrm{AQP} 4$ expression is virtually abolished in the core of damaged tissue after occlusion and reperfusion of the cerebral artery. AQP4 levels were also significantly reduced in the ischaemic penumbra, an area of affected tissue immediately adjacent to the anoxic core that, although threatened, can be rescued if blood flow is rapidly restored. Interestingly, $\alpha$-syntrophin levels were unchanged in these areas under the same conditions, indicating that the coupling between this adaptor molecule and AQP4 is sensitive to ischaemia and reperfusion.

It is somewhat surprising that the expression of a water channel that has the potential to hasten the reduction of swelling by facilitating the efflux of water should be decreased in response to the very cause of this damaging oedema. The authors postulate that this downregulation of AQP4 might occur during the early stages of ischaemia as a means of limiting the initial influx of water, thereby keeping swelling to a minimum. AQP4 could therefore be a useful target for the development of drug therapies that aim to minimise cerebral oedema. But will the extent by which swelling is minimised by such treatments compensate for the inevitable prolongation of the recovery period? This physiological dilemma - a consequence of the capacity of AQP4 to facilitate both the influx and efflux of water - will need to be taken into consideration if the expression of this regulatory water channel is to be therapeutically manipulated.

Suzanne Farley

(D) References and links

ORIGINAL RESEARCH PAPER Amiry-Moghaddam, M. et al. An $\alpha$-syntrophin-dependent pool of AQP4 in astroglial end-feet confers bidirectional water flow between blood and brain. Proc. Natl Acad. SCi. USA 100, 2106-2111 (2003)

Encyclopedia of Life Sciences: http://www.els.net stroke | water channels 